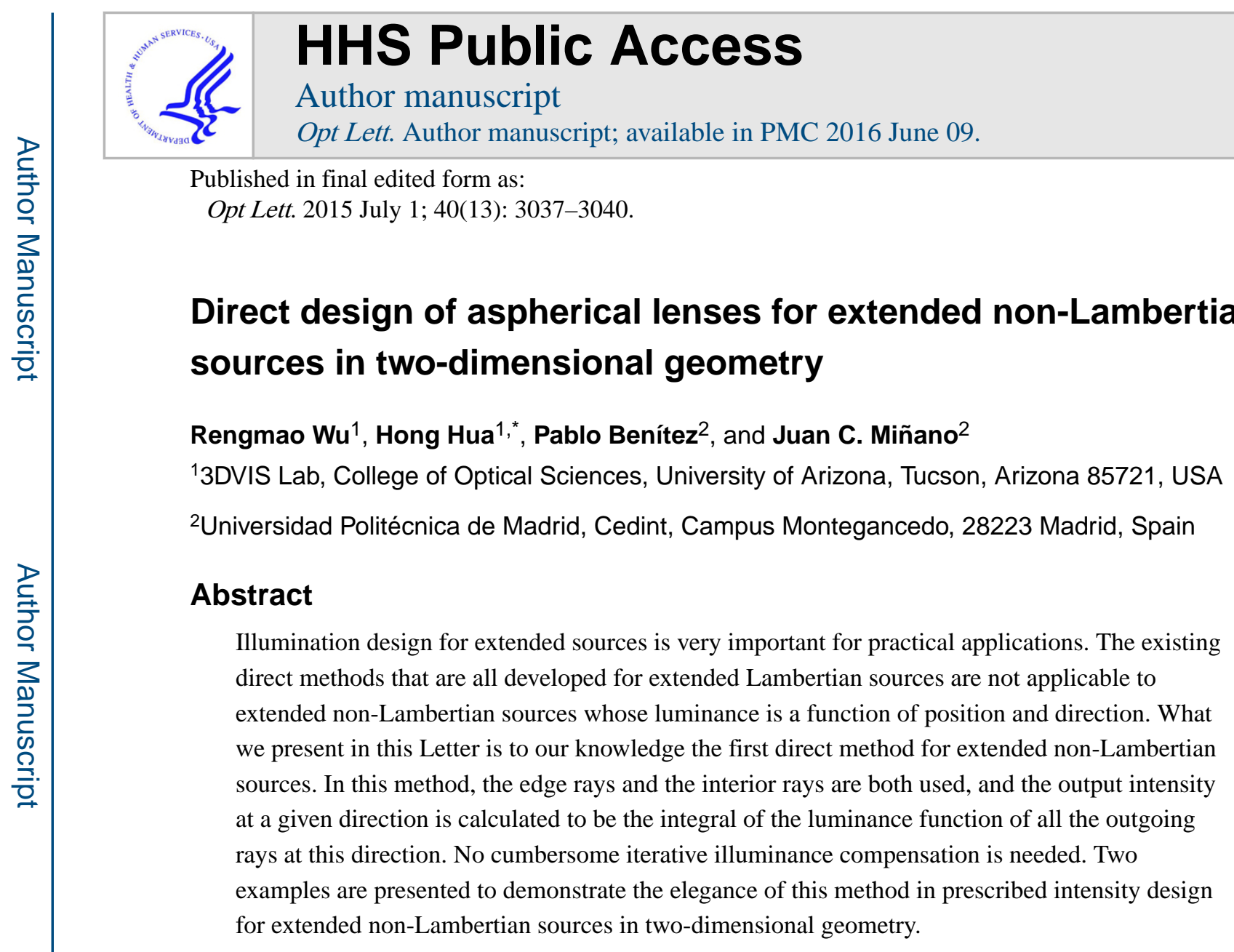

\title{
OCIS codes
}

(220.2945) Illumination design; (220.1250) Aspherics; (220.2740) Geometric optical design

\begin{abstract}
Direct application of light sources usually cannot meet the prescribed lighting requirements, and one typical purpose of illumination design is to redistribute the spatial energy distribution of the light source to generate a prescribed illumination by a means of some elaborately designed optical surfaces $\left[{ }^{1}\right]$. In practical applications, it is difficult to find an ideal light source, because an actual source has a certain size and angular extent. And, usually those zero-étendue methods [2-6], which rely on point source or parallel beam assumption, are not able to generate good designs when the influence of the source size or angular extent on the performance of an illumination system cannot be ignored. Illumination design for ideal sources has already been widely studied [2-6]. For extended sources, however, it is still not well addressed and faces many unresolved challenges.

Illumination design for extended sources can be classified into four subsidiary sets: twodimensional (2D) design, translational design, rotational design, and freeform design in three-dimensional (3D) geometry, as shown in Fig. 1. Of these four different designs, the 2D design is a fundamental design, which means some basic principles established in the translational design can be applied to the other two designs. To solve this fundamental
\end{abstract}

\footnotetext{
*Corresponding author: hhua@optics.arizona.edu.
} 
design problem, several direct methods have been developed in the past two decades [ ${ }^{7}{ }^{10}$ ]. However, these methods are only effective for Lambertian sources whose luminance is a constant. The luminance distribution of non-Lambertian sources is usually a function of position and direction, and inevitably these methods developed for Lambertian sources are not applicable to extended non-Lambertian sources. Some illuminance-compensation approaches developed for the rotational (or freeform) designs $\left[11^{14}\right.$ ] can be applied to $2 \mathrm{D}$ designs. The key to these design methods is iteratively using illuminance compensation to improve the performance of a design created by a zero-étendue method during each iteration. These methods may not be the best choice due to the cumbersome iterative raytracing process, and sometimes energy waste caused by total internal reflection cannot be avoided due to the designs created by a zero-étendue method.

In this Letter, we will develop a direct method of illumination design for extended nonLambertian sources in 2D geometry. Direct design means no cumbersome iterative raytracing is needed. And the method developed here is to our knowledge the first direct method for extended non-Lambertian sources. Usually, a prescribed illuminance design can be converted into a prescribed intensity design as long as the influence of the lens size on the optical performance can be ignored. Thus, we only focus on the prescribed intensity design in this Letter. Let $L(x, \theta)$ denote the luminance function of an extended non-Lambertian source, and we suppose the luminance function, $L(x, \theta)$, is positive in the domain of definition. We further assume that the optical system is loss-less. Then, the luminance of the incident rays will be conserved in this loss-less illumination system. That means the luminance, $L(x, \theta)$, of the infinitesimal light beam, 1, emitted from the point $\mathrm{R}$ on the source will be equal to that of its outgoing beam 2, after refraction of the lens, as shown in Fig. 2. This is an important principle for solving the design problem of extended non-Lambertian sources, and we will show how to calculate the output intensity by using this principle. Assume the extended light source is surrounded by a medium of refractive index $n$. Let $d$ denote the width of the source, $S_{1} S_{2}$, and suppose the source has an angular range of emission between $\theta_{\min } \leq \theta \leq \theta_{\max }\left(\theta_{\min }=-\theta_{\max }\right)$. Then, the total flux emitted from the light source is given by

$$
\Phi_{1}=\int_{\theta_{\min }}^{\theta_{\max }} \int_{-d / 2}^{d / 2} n L(x, \theta) \cos \theta \mathrm{d} x \mathrm{~d} \theta
$$

We suppose $I_{t}(\beta)$ is the prescribed output intensity distribution with an angular range between $\beta_{\min } \leq \beta \leq \beta_{\max }$ (here, $\beta_{\min }=-\beta_{\max }$ ). The conservation law of energy says the total flux of the outgoing beam should be equal to that of the incident beam. That is, $\Phi_{1}$ should also satisfy

$$
\Phi_{1}=\int_{\beta_{\min }}^{\beta_{\max }} I_{t}(\beta) \mathrm{d} \beta
$$

Next, we will generate the prescribed output intensity by using the luminance conservation in this loss-less system. 
Due to the nature of an extended source, an initial patch is required before we start to design the lens profile. In Fig. 3(a), $C_{1} C_{2}$ represents the initial patch. Here, $C_{2}$ is a mirror point of $\mathrm{C}_{1}$ about the z-axis. It is required that the two edge rays, $\mathrm{S}_{1} \mathrm{C}_{1}$ and $\mathrm{S}_{2} \mathrm{C}_{2}$, should be refracted by the initial patch to take the resulting direction angle $\beta=0^{\circ}$. An edge ray emitted from $\mathrm{S}_{2}$ passes through an arbitrary point $\mathrm{P}_{i}$ on $\mathrm{C}_{1} \mathrm{C}_{2}$ and takes the resulting direction angle $\beta_{i}$. To guarantee a successful design, it is required that $\beta_{i-1}<\beta_{i}$. Here, $\beta_{i-1}$ is the direction angle of the outgoing ray at the previous point $\mathrm{P}_{i-1}$, as shown in Fig. 3(a). Suppose the direction angle, $\beta$, of the outgoing ray can be represented mathematically by a monotonically increasing function $\beta=f(x)$. That is, $d \beta l d x>0$ on $\mathrm{C}_{1} \mathrm{C}_{2}$. To achieve this goal, we define a set of data points on $\mathrm{C}_{1} \mathrm{C}_{2}$ and $\mathrm{S}_{1} \mathrm{~S}_{2}$, respectively, which are equally spaced along the $\mathrm{x}$-axis, and let the incident rays $\mathrm{Q}_{i} \mathrm{P}_{i}(i=0,1, \ldots, N)$ exit the initial patch toward direction $\beta=0^{\circ}$ $\left.{ }^{15}\right]$, as shown in Fig. 3(b). Then, we can get a set of outgoing rays that are parallel to the $\mathrm{z}$ axis between the two edge rays, 1 and 2 . Since the incident rays of these outgoing rays are predefined, the luminance of these outgoing rays is, of course, known. Since these outgoing rays are parallel to each other, the luminance of these outgoing rays between the two edge rays 1 and 2 can be represented as a function of the distance 1 , which denotes the distance between the outgoing ray $i$ and the edge ray 1, as shown in Fig. 3(b). Suppose the function satisfies $L_{0}=f_{0}(I)\left(0 \leq 1 \leq I_{0}\right.$. Here, $I_{0}$ denotes the distance between the two edge rays 1 and 2 ). Then, the output intensity at direction $\beta=0^{\circ}$ is the integral of the function $L_{0}=f_{0}(I)$ on the closed interval $\left[0,1_{0}\right]$, which is given by

$$
I(0)=\int_{0}^{l_{0}} f_{0}(l) \mathrm{d} l
$$

Let us assume that the $\mathrm{z}$-coordinate of $\mathrm{C}_{1}$ is predefined as the value of $h$, and then we only need to choose an appropriate value of the $\mathrm{x}$-coordinate for $\mathrm{C}_{1}$ such that the output intensity, $I(0)$, at direction $\beta=0^{\circ}$ equals the prescribed intensity, $I_{t}(0)$.

When the initial patch is obtained, we can calculate the rest of the lens profile. Take the calculation of point $\mathrm{P}_{N+1}$ as an example. Trace an edge ray, $\mathrm{S}_{2} \mathrm{P}_{1}$, and calculate the direction angle $\beta_{1}$ of its outgoing ray, the ray 1 , as shown in Fig. 3(c). Then, calculate a set of points $\mathrm{R}_{i}(i=2,3, \ldots, N)$ on $\mathrm{S}_{1} \mathrm{~S}_{2}$, which are the points such that the rays $\mathrm{R}_{i} \mathrm{P}_{i}(i=2,3, \ldots, N)$ exit the initial patch also toward direction $\beta=\beta_{1}$. Since we have obtained the $\mathrm{x}$-coordinates of points $\mathrm{R}_{i}$, the luminance of these outgoing rays can be calculated. Also trace a ray from $\mathrm{S}_{1}$, and calculate the new point $\mathrm{P}_{N+1}$ of the lens profile as the intersection point between the ray $\mathrm{S}_{1} \mathrm{P}_{N+1}$ and the tangent line of its previous point $\mathrm{C}_{1}\left(\mathrm{P}_{N}\right)$, as shown in Fig. 3(c). Suppose this ray exits the lens also toward direction $\beta=\beta_{1}$. Obviously, the luminance of its outgoing ray, the ray 2 , is known. Then, we get a set of outgoing rays that are parallel to the two edge rays 1 and 2, and similarly the luminance of these outgoing rays between the two edge rays 1 and 2 can be represented as a function of the distance 1 . Assume this function satisfies $L_{1}=f_{1}(I)$. Similarly, the output intensity $I\left(\beta_{1}\right)$ at direction $\beta=\beta_{1}$ is the integral of the function $L_{1}=f$ ${ }_{1}(I)$ on the distance between the two edge rays 1 and 2 shown in Fig. 3(c). What we need to do next is only to adjust the position of the point $\mathrm{P}_{N+1}$ on the tangent line of the point $\mathrm{C}_{1}$ so that the output intensity $I\left(\beta_{1}\right)$ equals the prescribed intensity, $I_{t}\left(\beta_{1}\right)$. After that, we calculate the normal vector $\mathbf{N}_{N+1}$ by application of the Snell's law, and then perform the whole 
calculation described above until the direction angle of the incident ray from $\mathrm{S}_{1}$ satisfies $\theta$ > $\theta_{\max }$. In Fig. 3(d), $M$ is the right end point of the lens profile. Assume the direction angle of the incident ray $\mathrm{S}_{1} M$ equals $\theta_{\max }$, and that of its outgoing ray, the ray 6 , is $\beta_{m}$. We calculate the incident ray $\mathrm{S}_{2} \mathrm{P}_{j}$ with a direction angle $\theta_{j}$, which exits the lens also toward direction $\beta=$ $\beta_{m}$. Since only one surface is used here, we have $\theta_{j}<\theta_{\max }$ with no more degrees of freedom to control those rays emitted from $\mathrm{S}_{2}$ between $\theta_{j}<\theta \leq \theta_{\max }\left[{ }^{1}\right]$. It means some incident rays from $\mathrm{S}_{2}$ cannot be well controlled, and consequently we have $\beta_{m}<\beta_{\max }$ (here, we define $\beta_{m}$ as the maximum effective angle we can obtain). Then, an arbitrary ray emitted from $\mathrm{S}_{2}$ with a direction angle between $\theta_{j}<\theta \leq \theta_{\max }$ will take a resulting direction angle between $\beta_{m}<\beta$ $\leq \beta_{k}\left(\beta_{k}\right.$ is the direction angle of the ray 7. Usually, $\left.\beta_{k}>\beta_{\max }\right)$. Next, two examples will be given to verify the design method.

In the first example, the luminance distribution of the extended non-Lambertian light source and the prescribed output intensity distribution are both given in Eq. (4):

$$
\left\{\begin{array}{c}
L(x, \theta)=\cos \theta,\left\{(x, \theta) \mid-d / 2 \leq x \leq d / 2, \theta_{\min } \leq \theta \leq \theta_{\max }\right\} \\
I_{t}(\beta)=K_{1}, \beta \in\left[\beta_{\min }, \beta_{\max }\right]
\end{array}\right.
$$

The value of $K_{1}$ can be calculated by applying the energy conservation given in Eqs. (1) and (2), and the other design parameters are listed in Table 1. From Eq. (4), we can see that the luminance of the extended source is space-invariant. The normalized intensity distribution and the normalized luminance distribution of the light source are both given in Fig. 4(a). According to the method presented above, we also get the luminance distribution of the outgoing beam at directions $\beta=0^{\circ}$ and $\beta=40^{\circ}$, as shown in Figs. 4(b) and 4(c), respectively. From these two figures, we can see that the luminance distribution of the outgoing beam is quite different at different directions. Figure 4(d) gives the actual intensity distribution that is represented by the red solid line.

The fractional RMS is employed here to quantify the difference between the actual intensity and the prescribed one, which is defined by

$$
\mathrm{RMS}=\sqrt{\frac{1}{M_{1}} \sum_{k=1}^{M_{1}}\left(\frac{I_{a k}-I_{t k}}{I_{t k}}\right)^{2}}
$$

where $M_{1}$ is the number of the sample points, $I_{t k}$ is the target intensity of the k-th point, and $I_{a k}$ is the actual intensity of the k-th point. A smaller value of RMS represents less difference (of course, a better agreement) between the actual intensity and the prescribed one. Due to the limitation of one single surface, inevitably there will be a region of abrupt intensity change near $\beta_{\mathrm{C}}$, which denotes the maximum effective angle obtained from raytracing, as shown in Fig. 4(d). Since this region cannot be eliminated, a design can be considered acceptable as long as good agreement is achieved for the region within $\left[0, \beta_{\mathrm{C}}\right]$. According to the actual intensity distribution, we have $\beta_{\mathrm{C}}=53.23^{\circ}$ and $\mathrm{RMS}=0.0032$. Obviously, good agreement is achieved between the actual intensity and the target. The lens 
profile is given in Fig. 5(a). Let $H$ denote the z-coordinate of the vertex of the lens. From Fig. 5(a), we have $H / d=2.52$.

The second design is a more general case, in which the luminance of the extended nonLambertian source is a function of position and direction, as shown in Eq. (6):

$$
\left\{\begin{array}{c}
L(x, \theta)=\frac{\exp \left(-2 x^{2}\right)}{\cos \theta},\left\{(x, \theta) \mid-\frac{d}{2} \leq x \leq \frac{d}{2}, \theta_{\min } \leq \theta \leq \theta_{\max }\right\} \\
I_{t}(\beta)=\frac{K_{2}}{\cos \beta}, \beta \in\left[\beta_{\min }, \beta_{\max }\right]
\end{array}\right.
$$

Here, the value of $K_{2}$ can also be calculated by applying the energy conservation. Figure 6(a) gives the luminance distribution of the light source at the direction $\theta=0^{\circ}$, which is a Gaussian distribution with a beam waist of $1 \mathrm{~mm}$. Figures 6(b) and 6(c) show the luminance distribution of the outgoing beam at directions $\beta=0^{\circ}$ and $\beta=40^{\circ}$, respectively. The actual output intensity distribution is given in Fig. 6(d). From Fig. 6(d), we have $\beta_{\mathrm{C}}=52.88^{\circ}$ and RMS $=0.0021$. Obviously, the actual design is pretty good with little difference between the actual intensity and the prescribed one. The lens profile of this design is given in Fig. 5(b). From this figure, we can see the lens is quite compact, and we have $H / d=2.51$. The two examples both tell us that the proposed method is quite effective, and the two prescribed designs are both achieved successfully.

This Letter develops a direct design of aspherical lenses to solve the prescribed intensity problem for extended non-Lambertian sources in 2D geometry. In this method, we show how to calculate the output intensity by using the luminance of an extended non-Lambertian source, which is a function of position and direction. The examples perfectly show the elegance of this method in prescribed intensity design. However, we still need to point out that the convergence of the proposed method may not be guaranteed if $L(x, \theta)$ is not a strictly positive function in the domain of definition. For a 3D translationally refractive design, the aspherical lens can be generated by translating the lens profile at a given direction that is perpendicular to the meridian plane. As a result, the prescribed intensity can be achieved only in the meridian plane of the lens due to skew rays. However, this direct method can be fully applicable in 3D translationally reflective designs, and sometimes designs for skew rays can be also made to improve the $3 \mathrm{D}$ performance $\left[{ }^{16}\right]$. Although we only address the 2D design here, the proposed method could still be a huge step toward a practical and effective method for extended non-Lambertian sources. And, we will generalize this method to 3D rotational design for extended non-Lambertian sources in our future work.

\section{Acknowledgments}

National Institute of Biomedical Imaging and Bioengineering (NIBIB) (1R01EB18921-01).

\section{References}

1. Winston, R.; Miñano, JC.; Benítez, P. Nonimaging Optics. Elsevier; 2005.

2. Ries H, Muschaweck J. J Opt Soc Am A. 2002; 19:590.

3. Ding Y, Liu X, Zheng ZR, Gu PF. Opt Express. 2008; 16:12958. [PubMed: 18711534] 
4. Fournier FR, Cassarly WJ, Rolland JP. Opt Express. 2010; 18:5295. [PubMed: 20389542]

5. Bruneton A, Bäuerle A, Wester R, Stollenwerk J, Loosen P. Opt Express. 2013; 21:10563. [PubMed: 23669912]

6. Wu RM, Xu L, Liu P, Zhang YQ, Zheng ZR, Li HF, Liu X. Opt Lett. 2013; 38:229. [PubMed: 23454971]

7. Winston R, Ries H. J Opt Soc Am A. 1993; 10:1902.

8. Rabl A, Gordon JM. Appl Opt. 1994; 33:6012. [PubMed: 20936014]

9. Ong PT, Gordon JM, Rabl A, Cai W. Opt Eng. 1995; 134:1726.

10. Hernandez, M. PhD dissertation 04-030. Universidad Politécnica de Madrid; 2003. Development of nonimaging optical concentrators.

11. Bortz J, Shatz N. Proc SPIE. 2007; 6670:66700A.

12. Cassarly WJ. Proc SPIE. 2010; 7652:76522L.

13. Luo Y, Feng ZX, Han YJ, Li HT. Opt Express. 2010; 18:9055. [PubMed: 20588752]

14. Wester R, Müller G, Völl A, Berens M, Stollenwerk J, Loosen P. Opt Express. 2014; 22:A552. [PubMed: 24922264]

15. Wu RM, Qin Y, Hua H, Meuret Y, Benítez P, Miñano JC. Opt Lett. 2015; 40:2130. [PubMed: 25927803]

16. Minano JC. Appl Opt. 1984; 23:2017. [PubMed: 18212943] 


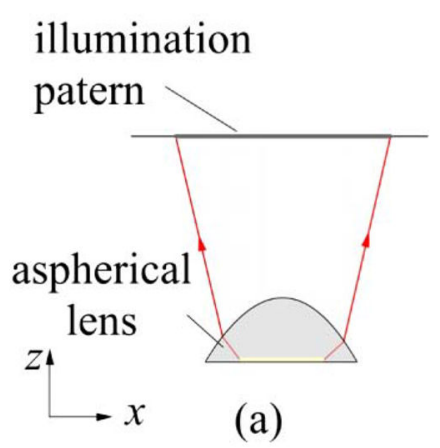

illumination

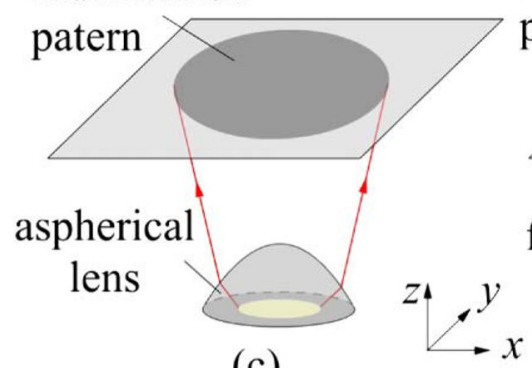

(c)

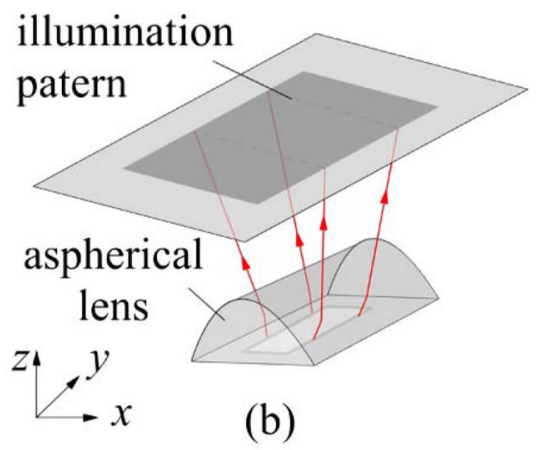

illumination

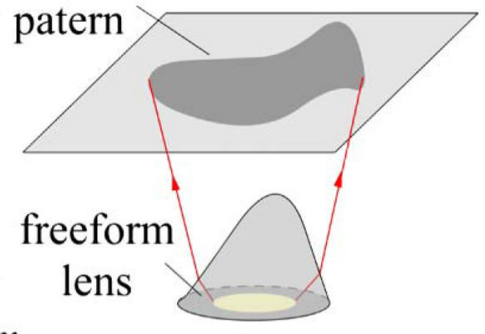

(d)

Fig. 1.

Illumination design for extended sources. (a) 2D designs are in any case first step toward a generalization for 3D-rotational or freeform systems. (b) In a translational design, the lens is created by translating the lens profile along the y-axis. (c) In a rotational design, the lens is created by applying the rotation to the lens profile. (d) In a freeform design, the lens has no rotational symmetry. 


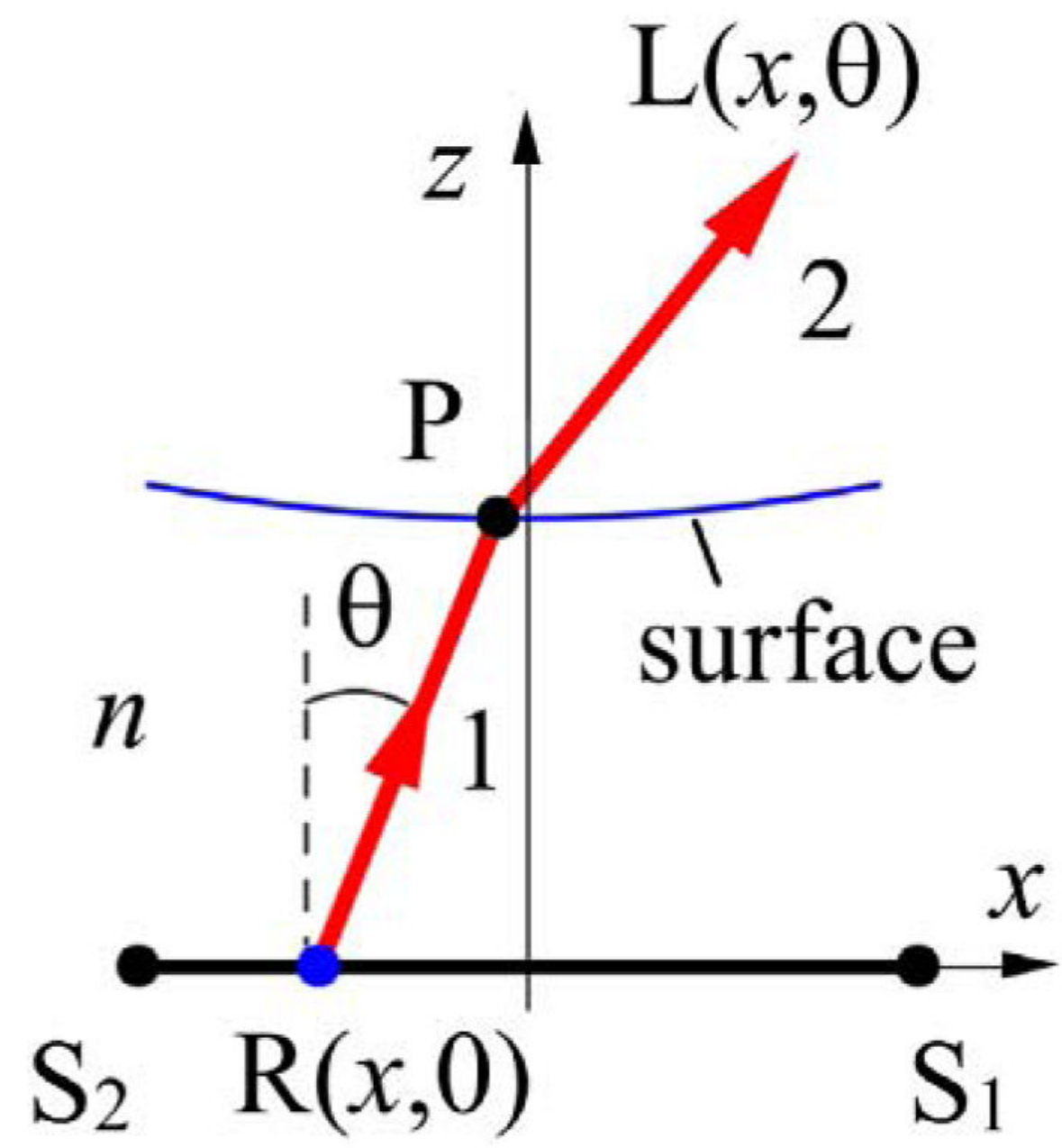

Fig. 2.

Luminance of an infinitesimal beam is conserved in a loss-less system. 


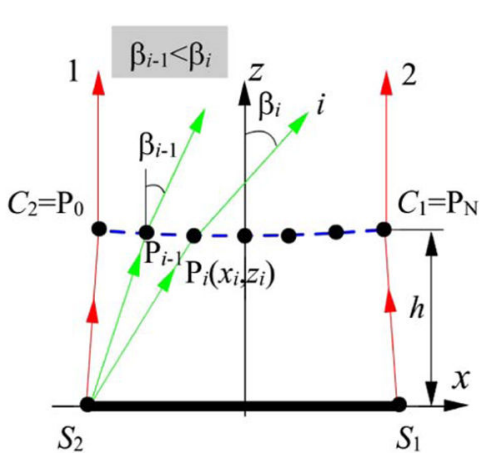

(a)

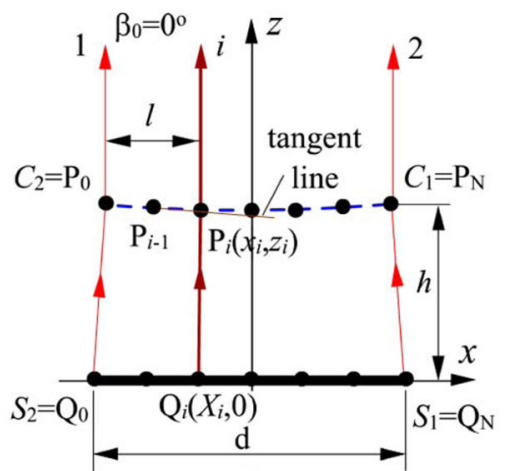

(b)

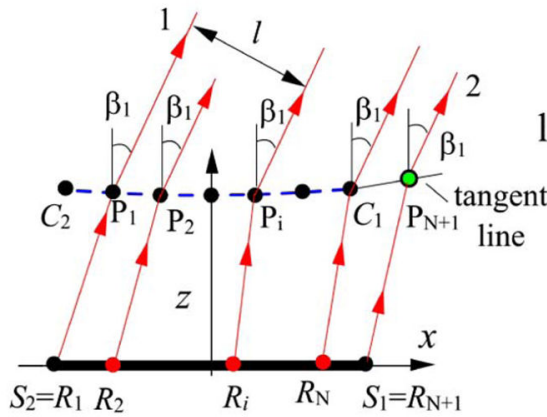

(c)

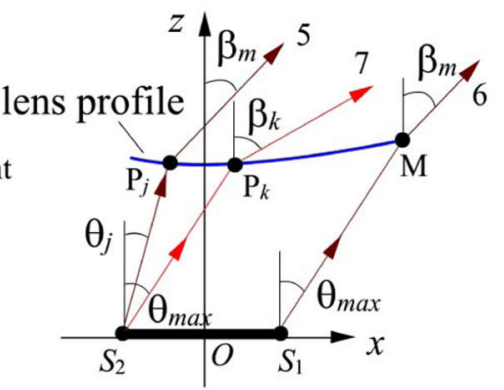

(d)

Fig. 3.

(a) $\beta=f(x)$ must be a monotone increasing function. (b) Calculate the initial patch and (c) calculate the rest of the lens profile. (d) Some specific considerations. Those rays from $S_{2}$ between $\theta_{j} \leq \theta \leq \theta_{\max }$ cannot be well controlled. 


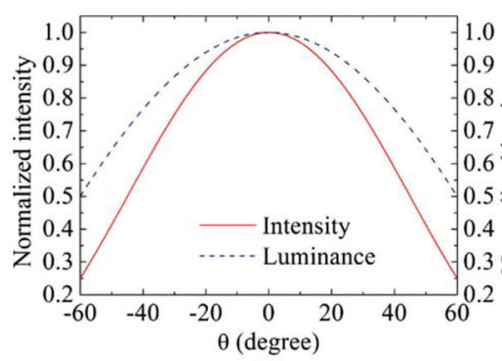

(a)

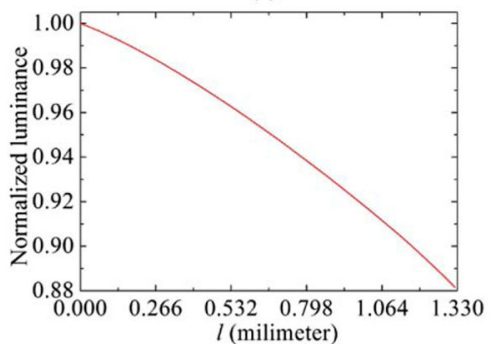

(c)

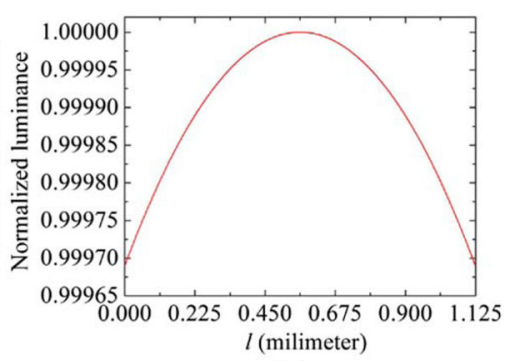

(b)

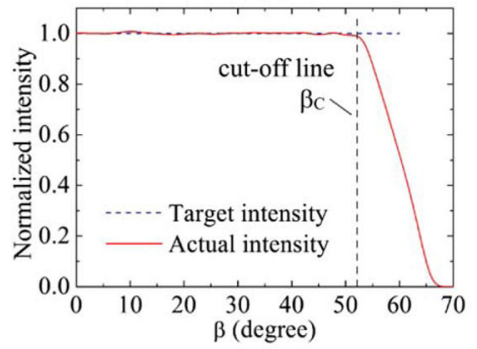

(d)

Fig. 4.

(a) The normalized intensity and the normalized luminance of the extended non-Lambertian source. (b) $L_{0}=f_{0}(I)$, the normalized luminance distribution of the outgoing beam at direction $\beta=0^{\circ}$ and (c) $L_{40}=f_{40}(I)$, the normalized luminance distribution of the outgoing beam at direction $\beta=40^{\circ}$. (d) The actual intensity and the prescribed intensity. 


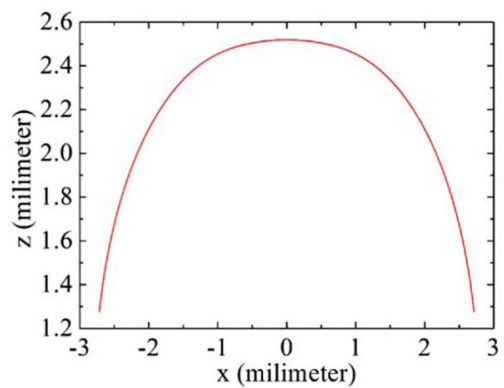

(a)

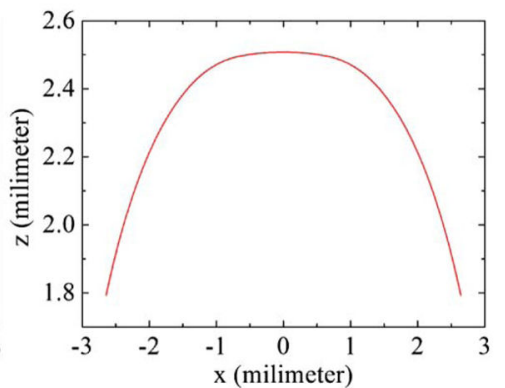

(b)

Fig. 5.

(a) The lens profile of the first design and (b) the lens profile of the second design. 


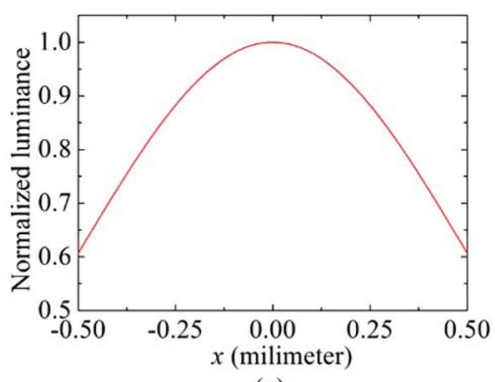

(a)

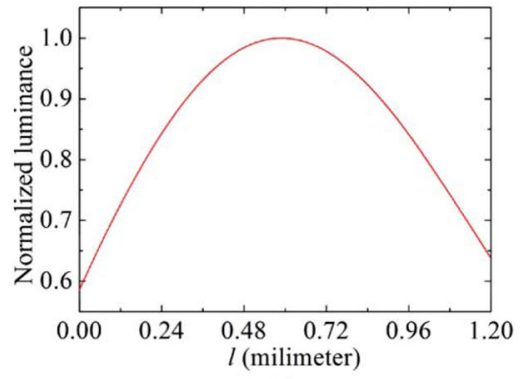

(c)

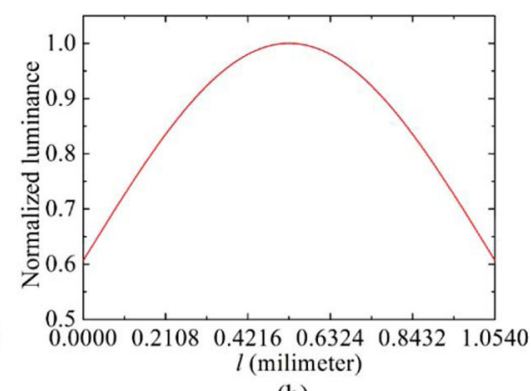

(b)

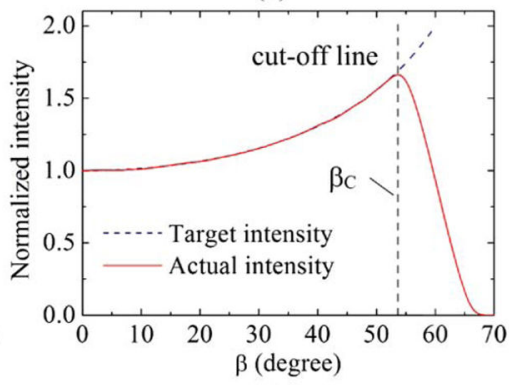

(d)

Fig. 6.

(a) The normalized luminance of the extended non-Lambertian light source at the direction $\theta$ $=0^{\circ}$. (b) $L_{0}=f_{0}(I)$, the normalized luminance distribution of the outgoing beam at $\beta=0^{\circ}$, and (c) $L_{40}=f_{40}(I)$, the normalized luminance distribution of the outgoing beam at $\beta=40^{\circ}$. (d) The actual intensity and the prescribed intensity. 

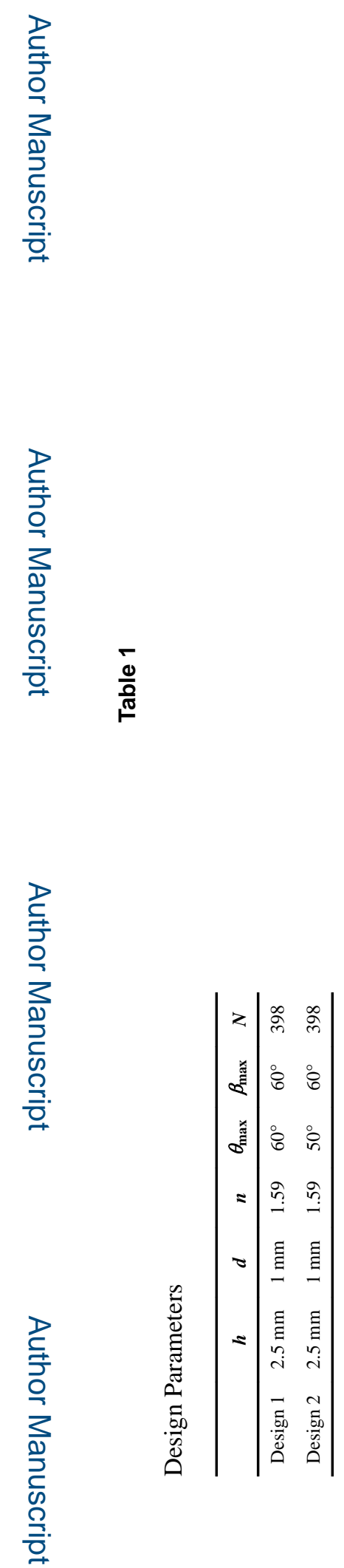

Opt Lett. Author manuscript; available in PMC 2016 June 09. 